\title{
Herbert Lochs, ehemaliger Rektor der Medizinischen Universität Innsbruck, ist am 10. Februar 2015 im 69. Lebensjahr verstorben
}

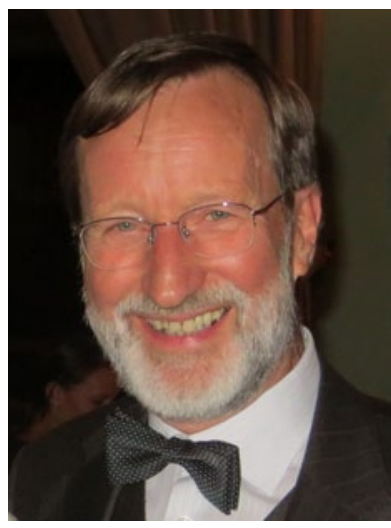

Herbert Lochs
Herbert Lochs stammt aus Innsbruck, wo er das Medizinstudium absolviert und die akademische Laufbahn am pharmakologischen Institut begonnen hat. 1973 kam er nach Wien an die I. Medizinische Universitätsklinik und wandte sich danach der Gastroenterologie und Hepatologie an der IV. Universitätsklinik zu. Hier entwickelte er seine Forschungsschwerpunkte: die entzündlichen Darmerkrankungen und die klinische Ernährung. Themen, die er in einem zweijährigen Studienaufenthalt an der Universität von Pittsburgh vertiefen konnte. Nach seiner Rückkehr nach Wien leitete er eine Arbeitsgruppe an der Gastroenterologie zum Thema Stoffwechsel und Ernährung. Neben seiner wissenschaftlichen Tätigkeit war er auch in universitären Belangen sehr engagiert. So prägte er neben seiner Tätigkeit in verschiedenen universitären Kommissionen auch als langjähriger Schriftführer der Ethikkommission nachhaltig deren Strukturen.

1994 wurde Herbert Lochs an die Charité in Berlin berufen und wirkte dort als Direktor der Medizinischen Klinik und Poliklinik mit Schwerpunkt Gastroenterologie, Hepatologie und Endokrinologie. Er wurde einer der anerkanntesten Gastroenterologen Deutschlands. Seine Expertise für entzündliche Darmerkrankungen war weltweit anerkannt. Herbert Lochs hat in Berlin eine „Schule der klinischen Ernährung“ begründet und diesen Fachbereich im deutschen Sprachraum grundlegend geformt. Unter seiner Ägide wurden zahlreiche wichtige Leitlinien der Deutschen Gesellschaft für Ernährungsmedizin (DGEM) verfasst, die später auch von der europäischen Gesellschaft übernommen wurden.

2009 kehrte Herbert Lochs als Rektor der Medizinischen Universität Innsbruck nach Österreich zurück, wo er auch nach der Beendigung seiner Amtsperiode blieb.
Herbert Lochs war ein kreativer Wissenschafter, hat zahlreiche nationale und internationale Projekte geführt und junge Wissenschafter gefördert. Ebenso wurden ihm zahlreiche Auszeichnungen zuteil, darunter das österreichische Ehrenkreuz für Wissenschaft und Kunst Erster Klasse.

Herbert Lochs war ein hingebungsvoller Arzt und engagierter Lehrer, dazu ein allseits gebildeter, kunstsinniger, vor allem der Musik verbundener Mensch. Sehr liebte er auch die Berge seiner Heimat Tirol. An der Seite der Gefährtin seines Lebens, seiner Frau Heide Hörtnagl, einer anerkannten Neuropharmakologin, hat sich das Leben von Herbert Lochs entfaltet. Bis zuletzt voll gegenwärtig hat er unter der hingebungsvollen Begleitung seiner Frau Heide seinem tückischen Leiden über Monate widerstanden.

Herbert Lochs hat zahlreiche wissenschaftliche Beiträge in der Wiener klinischen Wochenschrift veröffentlicht und war über mehr als zwei Jahrzehnte in ihrem Editorial Board. Mit ihm verlieren wir einen sehr ideenreichen Kollegen, einen konstruktiven Gutachter und einen guten Freund.

Univ.-Prof. Dr. Gerold Stanek Editor-in-Chief E-Mail: gerold.stanek@meduniwien.ac.at 